\title{
In the $60^{\text {th }}$ jubilee of Professors of Tomsk Polytechnic University Igor V. Minin and Oleg V. Minin
}

The guest editors represent this Special Issue of the Journal of Biomedical Photonics \& Engineering in the Honor $60^{\text {th }}$ jubilee of Profs. Igor V. Minin and Oleg V. Minin, DSc, Corr.-Ms of Russian Academy of Metrology and Russian Academy of Natural History who have made a significant contribution to the field of mesoscale diffraction optics and nanophotonics. Profs. I.V. Minin and O.V. Minin were born on the $22^{\text {nd }}$ of March in 1960 in Novosibirsk Academytown. They graduated from Novosibirsk State University in 1982. I.V. Minin defended his Ph.D. thesis at St. Petersburg Electrotechnical Institute in 1986 while O.V. Minin upheld his candidate dissertation at Tomsk Institute of Atmospheric Optics, in 1987. They defended their Dr.Sc. theses at the Novosibirsk State Technical University, in 2004. The brothers began their scientific work at the Institute of Applied Physics in Novosibirsk starting from Lab Assistants to Leading Researches. Now they are Professors at Tomsk Polytechnic University.

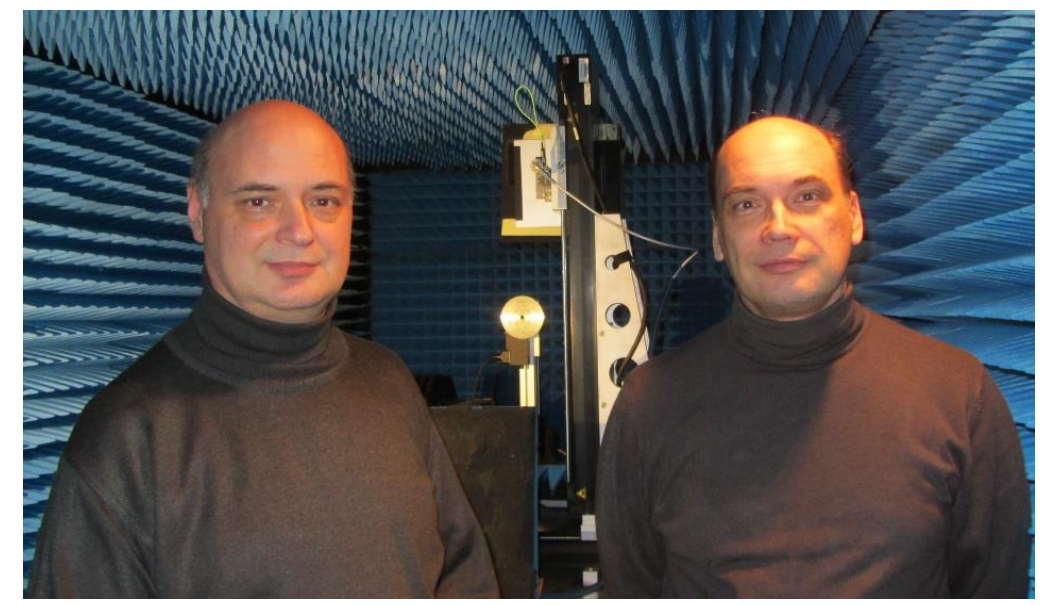

Professors of Tomsk Polytechnic University

Prof. Igor V. Minin (left) and Prof. Oleg V. Minin (right) standing beside the echoless chamber.

Profs. I.V. Minin and O.V. Minin have 300 joint research publications in peer-reviewed journals indexed in Web of Science and Scopus, more than 120 patents on invention, and 18 monographs. Among the monographs we should mention the well-known issues: "Diffractive optics of millimeter waves", IOP Publisher, London-Boston, in 2004; "Three Dimensional Fresnel Antennas", Research Signpost, Kerala, India, in 2005; "Basic Principles of Fresnel Antenna Arrays", Springer, in 2008, and "Diffractive Optics and Nanophotonics", Springer International Publishing, Cham, in 2016.

The biographical data of Profs. I.V. Minin and O.V. Minin is included in Marquis "Who's Who in the World" in 2003; Russian reference book "Scientists of Russia" in 2004; in the encyclopedia "Scientists of Russia", in 2008; "Who's Who in Russia" in 2010. They were awarded the diploma from the monthly published magazine Laser Focus World in 2003 for a series of publications in the field of telecommunication technologies and counter-terrorism. Profs. I.V. Minin and O.V. Minin hold the Certificate of Honor of the Ministry of Engineering and the Presidium of the Central 
Committee of the USSR Trade Union, and the Certificate of the Minister of Defense of the Russian Federation for their scientific contribution during 1997-2000.

Profs. I.V. Minin and O.V. Minin are currently the experts of the International Program COST 284 "Innovative Antennas for Emerging Terrestrial \& Space-based Applications" and IASTED as well. Also, they are the experts in different Federal programs and foundations as Russian Foundation for Basic Research, Russian Science Foundation. They both are the SPIE members, and have been the members of various International conferences and meetings from 2008-2020. Profs. I.V. Minin and O.V. Minin are the members of the Editorial board of several recognized journals.

For a long time, they have been the Invited Professors to many companies and Universities: DaimlerChrysler AG (Germany); Doiche Aerospace (Germany); Technische Universitat Munchen, Germany; Harbin Institute of Technology, China; Samsung Electronics, Korea; National University of Singapore; MMW State Key Lab, Nanjing, China; Universidad Tecnica Federico Santa Maria, Chile, NJUST; Bieijing Institute of Technology, China; Capital Normal University, China; CRC, Canada; Helsinki University, Finland, etc.

Profs. I.V. Minin and O.V. Minin have established strong research foundations in different branches of physics, having a global priority, such as shock wave focusing by diffractive optics, explosive plasma antennas, subwavelength structured light and hypercumulative shaped charge. They have developed new types of 3D diffraction lenses, antennas and antenna arrays for telecommunication systems and millimetre-wave and THz imaging systems. Their main interest in mesoscale photonics and plasmonics focuses on terajets, acoustic jets and plasmonic jets. They discovered numerically a new curved beam, a photonic hook, for free space, acoustics and surface plasmon waves, which are much easier to produce compared to the Airy-family beams. Recently, the photonic hook phenomenon has been experimentally verified.

This Special Issue in the honor $60^{\text {th }}$ jubilee of Profs. I.V. Minin and O.V. Minin provides a multidisciplinary reflection on their career, indicating some of their scientific interests and contributions.

The guest editors of this Special Issue would like to thank all authors for their efforts in preparing their contributions and thank all the reviewers for their help in reviewing these papers. Last but not least, we would like to thank the editorial staff of the Journal of Biomedical Photonics

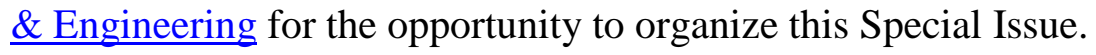

Special Issue Editors:

Kirill I. Zaytsev

Head of Laboratory of Broadband Dielectric Spectroscopy,

Prokhorov General Physics Institute of RAS, Moscow, Russia

E-mail: kirzay@gmail.com

Pavel F. Baranov

Head of division, National Research Tomsk Polytechnic University, Tomsk, Russia

E-mail: $\underline{\text { bpf@tpu.ru }}$

\section{Dmitry S. Ponomarev}

Deputy Director, V.G. Mokerov Institute of Ultra-High Frequency Semiconductor Electronics of RAS, Moscow, Russia

E-mail: ponomarev_dmitr@mail.ru 